\title{
ESTUDO DAS CONDIÇÕES FÍSICAS DA COLEÇÃO DE LIVROS DA ÁREA DE DIREITO: UM EXERCÍCIO DIDÁTICO
}

\section{ESTUDIO DE LAS CONDICIONES FÍSICAS DE LA COLECCIÓN DE LIBROS DEL ÁREA DE DERECHO: UN EJERCICIO DIDÁCTICO}

Maria Aparecida Lopes - maplopes@sercomtel.com.br Mestre em Ciência da Informação pela UNESP, Marília. Professora do Departamento de Ciência da Informação da Universidade Estadual de Londrina (UEL).

Maria Elisabete Catarino - beteca@uel.br Doutora em Tecnologias e Sistemas de Informação pela Universidade do Minho, Portugal. Professora adjunta do Departamento de Ciência da Informação da Universidade Estadual de Londrina (UEL).

Yara Maria Pereira da Costa Prazeres - yprazeres@sercomtel.com.br Mestre em Ciência da Informação pela Universidade de Campinas (Unicamp). Professora Aposentada do Departamento de Ciência da Informação da Universidade Estadual de Londrina (UEL).

Osny Terciotti - terciotti@uel.br Bibliotecário especialista em conservação de documentos do Sistema de Bibliotecas da Universidade Estadual de Londrina

(UEL).

\footnotetext{
RESUMO

Introdução: Relato das atividades práticas desenvolvidas com estudantes da Disciplina Preservação de Documentos (2BIB050) do Curso de Biblioteconomia da Universidade Estadual de Londrina (UEL), dentro do Programa de Formação Complementar no Ensino de Graduação por meio do projeto "A contribuição da gestão de bibliotecas universitárias para a formação do aluno de Biblioteconomia".

Objetivo: Avaliar o estado de conservação dos livros do curso de Direito alocados na Biblioteca Setorial de Ciências Humanas/BSCH da UEL.
} 
Maria Aparecida Lopes; Maria Elisabete Catarino; Yara Maria Pereira da Costa Prazeres; Osny Terciotti

Estudo das condições físicas da coleção de livros da área de Direito: um exercício didático

Metodologia: O exercício de diagnóstico foi realizado após as aulas teóricas sobre agentes de deterioração. Os estudantes foram encaminhados para a $\mathrm{BSCH}$ para analisar os livros das disciplinas selecionadas, tendo como instrumento uma ficha de avaliação adaptada do estudo de KRAMER.

Resultados: Os resultados parciais indicam que a maioria dos livros apresenta sujidades, além de danos nas capas e lombadas.

Conclusões: Conclui-se que as sujidades e danos identificados são provocados pelo manuseio incorreto e falta de higienização. Vale ressaltar que o bibliotecário, como responsável pela conservação e preservação dos acervos que gerencia, deve conhecer e pesquisar os fatores que provocam a deterioração dos materiais e as formas de combatê-los, a fim de estabelecer políticas e programas adequados à sua realidade.

Palavras-Chave: Diagnóstico de acervo. Conservação de coleções. Direito.

\section{INTRODUÇÃO}

A proposta deste texto é relatar as atividades de um Programa de Formação Complementar no Ensino de Graduação do Curso de Biblioteconomia da Universidade Estadual de Londrina (UEL), intitulado A contribuição da gestão de coleções de bibliotecas universitárias para a formação do aluno de Biblioteconomia (CATARINO, 2012).

O referido Programa está regulamentado na Universidade pela Resolução n. 0142/2012 do Conselho de Ensino Pesquisa e Extensão, definido como um "conjunto de ações de natureza acadêmica, social ou cultural que proporcionem a participação dos estudantes regularmente matriculados em cursos de graduação da Universidade, por meio de estudos e práticas complementares à formação curricular." (UNIVERSIDADE ESTADUAL DE LONDRINA, 2012).

Numa perspectiva multidisciplinar do ensino de Gestão de Bibliotecas, o Programa envolve, num primeiro momento, as disciplinas de Administração, Formação e Desenvolvimento de Coleções, Conservação e Preservação de Documentos e Estágios Curriculares do Curso de Biblioteconomia da UEL, com a participação dos estudantes das respectivas disciplinas.

Esse trabalho foi desenvolvido com os estudantes de Preservação de Documentos (2BIB050) com o objetivo de avaliar o 
Maria Aparecida Lopes; Maria Elisabete Catarino; Yara Maria Pereira da Costa Prazeres; Osny Terciotti

Estudo das condições físicas da coleção de livros da área de Direito: um exercício didático

estado de conservação dos livros das disciplinas Direito Penal, Direito Processual Penal, Direito do Trabalho e Processo do Trabalho, que fazem parte da bibliografia básica de 13 disciplinas do Curso de Direito da Universidade Estadual de Londrina, alocados na Biblioteca Setorial de Ciências Humanas.

O texto foi organizado situando algumas questões referentes à gestão de acervos, com ênfase na preservação e conservação de documentos. Em seguida, apresenta conceitos e um breve referencial sobre a área de conservação de materiais bibliográficos. Na sequência, discorre sobre a metodologia utilizada e as ações desenvolvidas pelos estudantes como atividade prática da referida disciplina.

\section{GESTÃO DE ACERVOS}

A biblioteca universitária tem como função primordial atender às necessidades de informação do ensino de graduação e pós-graduação e dos programas de pesquisa e extensão desenvolvidos na comunidade universitária.

Para cumprir adequadamente seus objetivos, reúne, armazena e promove o acesso a uma grande quantidade de recursos informacionais, que constituem o patrimônio cultural da instituição.

A gestão dos acervos sob sua custódia faz parte integrante do planejamento da biblioteca, envolvendo a formação e o desenvolvimento da coleção, de acordo com as necessidades dos seus usuários, a conservação dos materiais incorporados à coleção e à avaliação desses recursos de informação em conformidade com as políticas estabelecidas nesse processo.

As atividades de preservação e conservação são, portanto, consideradas uma das funções de gerenciamento das coleções com vistas à promoção da integridade e longevidade dos registros da informação. Devem ser reconhecidas como medidas administrativas que visam à preservação dos bens culturais da instituição, a garantia do 
Maria Aparecida Lopes; Maria Elisabete Catarino; Yara Maria Pereira da Costa Prazeres; Osny Terciotti

Estudo das condições físicas da coleção de livros da área de Direito: um exercício didático

acesso à informação às gerações atuais e futuras e a redução de custos devido à escassez dos recursos orçamentários.

A implementação de ações para salvaguardar o acervo documental requer um planejamento que auxilie a tomada de decisões por meio da formulação de diretrizes e medidas a serem adotadas para a preservação dos documentos. Esse planejamento só é viável a partir do levantamento de dados qualitativos e quantitativos sobre as condições e necessidades de preservação, por meio de metodologias específicas para a coleta e análise dos dados que possibilitem 0 estabelecimento de prioridades e a racionalização de custos para sua execução (BOJANOSKI, 2012).

É imprescindível que os profissionais responsáveis pelo gerenciamento do acervo conheçam e procurem identificar os fatores intrínsecos e extrínsecos de deterioração dos documentos. No primeiro caso, os danos podem ser decorrentes dos materiais e processos utilizados na sua fabricação. No outro caso, podem ser causados pelas condições ambientais e de guarda dos documentos.

Sendo assim, o trabalho de diagnóstico do estado de conservação representa um procedimento metodológico importante para o conhecimento e mapeamento da situação, visando à solução dos problemas detectados. É fundamental também para auxiliar na execução de um cronograma de atividades, decisivo para a definição de prioridades da equipe de trabalho e dos custos para sua execução (MAGUETA, 2012).

Nesse processo, o levantamento dos dados sobre o estado de conservação dos acervos deve ser um dos primeiros passos para a tomada de decisões gerenciais. Tem como objetivo avaliar as condições físicas das obras visando a estabelecer critérios para a manutenção de coleções importantes no campo científico, didático e cultural de interesse para a biblioteca universitária, para a restauração, desbastamento ou descarte de materiais. 
Maria Aparecida Lopes; Maria Elisabete Catarino; Yara Maria Pereira da Costa Prazeres; Osny Terciotti

Estudo das condições físicas da coleção de livros da área de Direito: um exercício didático

Por meio dessa análise é possível identificar itens que necessitam de tratamento emergencial, obras danificadas pelo manuseio inadequado, tipos de danos sofridos, reparos a serem desenvolvidos, bem como material sem condições de recuperação ou cujo uso não justifique uma intervenção de tratamento (restauração ou encadernação). O resultado dessa análise contribui para a formulação de uma política de preservação de acervos que contemple as ações preventivas necessárias bem como a adoção de procedimentos adequados para a conservação das coleções.

\section{PRESERVAÇÃO E CONSERVAÇÃO DE ACERVOS}

Para melhor entendimento sobre Preservação, Conservação e Restauração, torna-se necessário apresentar alguns conceitos esclarecedores.

Para Conway (2001, p. 14), "Preservação é a aquisição, organização e distribuição de recursos a fim de que venham a impedir posterior deterioração ou renovar a possibilidade de utilização de um seleto grupo de materiais."

Stocker (2008, p. 53) afirma que é necessário diferenciar os processos de preservação e conservação. Para a autora, "Preservar supõe ações para reter os itens potenciais que podem causar danos nas obras, ou seja, resguardar o bem para que ele não sofra nenhum mal ou dano", enquanto "Conservar é o conjunto de ações dirigidas para alongar a vida da obra, é resguardar de dano, de decadência, deterioração etc."

No entendimento de Carvalho,

Por Restauração compreendem-se (sic) as intervenções técnicas sobre os componentes materiais e morfológicos de um documento já deteriorado, praticadas por especialistas em laboratórios, com o propósito de recuperá-lo para integridade estética e 
Maria Aparecida Lopes; Maria Elisabete Catarino; Yara Maria Pereira da Costa Prazeres; Osny Terciotti

Estudo das condições físicas da coleção de livros da área de Direito: um exercício didático

histórica da peça. (CARVALHO apud MOTTA; REIS, 2012, p. 37).

Conforme assinala Beck (1997, p. 5), preservação "[...] é toda ação que se direciona à salvaguarda das condições físicas dos materiais." A autora conceitua conservação/restauração como "intervenção na estrutura dos materiais, visando melhorar o seu estado físico". Sobre conservação preventiva, afirma que é a "melhoria do meio ambiente e dos meios de armazenagem, visando prevenir e retardar a degradação."

Neste contexto, podemos dizer que Restauração é uma interferência direta no documento danificado, visando a, na medida do possível, manter a sua forma original. Conservação desenvolve ações de rotina, objetivando retardar o processo de degradação dos documentos, enquanto a Preservação busca o planejamento das ações de restauração, estabelecendo políticas, elaborando projetos, procurando manter as obras em condições de manuseio para atender a demanda do usuário.

São muitos os fatores que contribuem para o processo de deterioração dos materiais bibliográficos.

Para Motta e Reis (2012, p. 37),

[...] é preciso destacar os elementos que atuam na deterioração dos acervos, [...] os agentes danificadores dos livros em termos de sua vida útil podem ser de várias naturezas, tais como a má qualidade da matéria prima e dos processos com que foram confeccionados como papel, colas e tintas.

Estes são fatores considerados intrínsecos, pois ocorrem durante o processo de fabricação do papel, devido à utilização de fibras de má qualidade, água impura e produtos químicos para o branqueamento da polpa e as colas. 
Maria Aparecida Lopes; Maria Elisabete Catarino; Yara Maria Pereira da Costa Prazeres; Osny Terciotti

Estudo das condições físicas da coleção de livros da área de Direito: um exercício didático

Segundo Silva (2012, p. 96), "O dano causado [...] depende das características químicas destas substâncias que podem apresentar incompatibilidade química entre si.", causando como consequência a acidez do papel e a oxidação das fibras, danos identificados por manchas com aparência de ferrugem.

Fatores considerados externos também têm influência na degradação dos suportes documentais, como a poeira e a poluição atmosférica; iluminação, temperatura e umidade relativa; incêndios, inundações; insetos e roedores; manuseio e armazenamento incorretos das obras, causados pelo próprio homem.

$\mathrm{O}$ acúmulo de poeira sobre os livros e documentos facilita o desenvolvimento de fungos, pois a poeira é composta de esporos de micro-organismos. Por ser abrasiva, provoca nos papéis um desgaste em sua superfície, tornando-os fragilizados. A poluição atmosférica também provoca a deterioração dos acervos. Spinelli Junior (1997, p. 27) afirma que "A atmosfera pode ser considerada um grande recipiente onde, permanentemente, são lançados sólidos, líquidos e gases capazes de comprometer a integridade dos acervos documentais."

A iluminação natural ou artificial prejudica os documentos em razão de possuírem raios ultravioleta que aceleram a degradação da celulose, deixando os papéis fragilizados. Para Ogden (2001b, p. 13), "A luz pode causar $\mathrm{O}$ descoloramento, $\mathrm{O}$ amarelecimento ou $\mathrm{O}$ escurecimento do papel, e pode enfraquecer e enrijecer as fibras da celulose que o compõem."

A falta de controle da temperatura e umidade relativa nos ambientes de guarda influencia na deterioração dos suportes documentais, reduzindo a sua vida útil. No entendimento de Silva (2012, p. 98), "[...] uma temperatura constante de armazenamento de $20^{\circ} \mathrm{C}$ é considerada adequada, sendo confortável aos trabalhadores e baixa o suficiente para não danificar os materiais." Quando em excesso, a temperatura acelera $\mathrm{o}$ processo de degradação dos livros e documentos. 
Maria Aparecida Lopes; Maria Elisabete Catarino; Yara Maria Pereira da Costa Prazeres; Osny Terciotti

Estudo das condições físicas da coleção de livros da área de Direito: um exercício didático

Por serem higroscópicos, os papéis absorvem a umidade, facilitando o desenvolvimento de fungos, bactérias e manchas. As grandes oscilações de umidade relativa provocam nas fibras de celulose um movimento de contração e dilatação, tornando os papéis fracos e quebradiços. Segundo Ogden (2001c, p. 7), "A instalação de controles climáticos adequados e a sua operação no sentido de manter os padrões de conservação retardarão consideravelmente a deterioração dos materiais."

A água e o fogo também provocam grandes danos aos documentos. Um pequeno vazamento no encanamento, obstrução de calhas e ralos, infiltrações nas paredes, enchentes, entre outros, podem molhar parte do acervo, ocasionando danos que muitas vezes são extensos.

O fogo, na maioria dos casos, destrói completamente os suportes da informação. Caso os documentos não sejam atingidos diretamente pelas chamas, o calor e a fuligem também danificam os suportes.

Da mesma forma, agentes biológicos, como microrganismos, insetos e roedores contribuem, em grande escala, na deterioração dos livros e documentos.

Fungos e bactérias contaminam o acervo contribuindo para a sua degradação. A poeira é um veículo para os esporos de microrganismos, que se depositam na superfície dos livros, aguardando condições propícias para se desenvolver, quais sejam: temperatura e umidade relativa do ambiente de guarda elevadas e falta de circulação de ar. Os fungos podem ser identificados por manchas de diversas cores, e uma aparência porosa do papel.

Os insetos vêm destruindo coleções importantes em acervos e são atraídos por restos de alimentos deixados nos locais de guarda e falta de controle de temperatura e umidade. Outros fatores que contribuem para os atrair são as substâncias orgânicas encontradas nos papéis, colas e couro das capas. 
Maria Aparecida Lopes; Maria Elisabete Catarino; Yara Maria Pereira da Costa Prazeres; Osny Terciotti

Estudo das condições físicas da coleção de livros da área de Direito: um exercício didático

Dentre os insetos bibliófagos mais destruidores de acervos estão a broca e o cupim. No entender de Stocker (2008, p. 58), “As brocas [...] atacam os documentos em sua fase de vida larvária, sendo por isso um dos mais nocivos." As larvas desenvolvem-se a partir das lombadas de livros bem fechados e apertados nas estantes, o que facilita a sua locomoção. No seu caminho, perfuram as folhas em busca de uma saída. $\mathrm{O}$ ataque das brocas pode ser identificado por meio dos furos no livro e de uma poeira fina encontrada nas estantes, sob o dorso de cada livro.

Da mesma forma que as brocas, os cupins também são muito agressivos quando se trata de ataque ao acervo. Alimentam-se da celulose; em razão disso atacam os papéis, livros e estruturas de madeira; atacam os materiais de forma rápida, destruindo quase todo o miolo dos livros.

Além das brocas e cupins, outros insetos, como baratas e traças contribuem para a deterioração do acervo.

As baratas são atraídas por restos de alimentos e pelas colas das encadernações. Geralmente atacam no período noturno, produzindo excrementos que mancham os papéis, deixando ranhuras ou trilhas disformes na superfície das folhas.

Em se tratando de destruição de acervos, os roedores, além de destruir os papéis, atacam os revestimentos dos condutores elétricos ocasionando incêndios.

Nas palavras de Silva (2012, p. 101), "O humano, ao lado dos insetos e microrganismos, é outro ser vivo que pode tornar-se um 'inimigo' dos livros e documentos."

Pela falta de cuidados no manuseio e armazenamento, o homem provoca danos físicos no material bibliográfico.

O uso de marcadores de páginas confeccionados com papéis de baixa qualidade; o suor das mãos em contato com o papel; o consumo de alimentos e bebidas durante o manuseio de livros e documentos; o uso de clipes metálicos; umedecer os dedos na saliva para virar as 
Maria Aparecida Lopes; Maria Elisabete Catarino; Yara Maria Pereira da Costa Prazeres; Osny Terciotti

Estudo das condições físicas da coleção de livros da área de Direito: um exercício didático

páginas; rasgos; rabiscos; subtração de páginas e capítulos são ações que contribuem para a destruição do material bibliográfico.

Segundo Ogden (2001a, p. 7), "O armazenamento inadequado tem efeito direto sobre a vida útil dos materiais." O uso de bibliocantos é indicado para manter os livros bem posicionados nas estantes, enquanto os livros de grande formato devem ser armazenados na posição horizontal, evitando, assim, que as folhas se descolem da lombada.

Os materiais de grandes dimensões, como mapas, plantas, cartazes e desenhos arquitetônicos devem ser armazenados em mapotecas, evitando serem dobrados, diminuindo o risco de danos.

Vale ressaltar que todos os fatores extrínsecos de deterioração podem ser eficientemente controlados por meio da implantação de um programa de conservação preventiva. O programa pode ser iniciado com procedimentos básicos referentes às condições ambientais favoráveis aos acervos documentais, representados pela higienização periódica, controle da temperatura, da umidade relativa, da iluminação, do acondicionamento adequado dos materiais, além de ações educativas voltadas para a conscientização de funcionários e usuários do sistema de informação.

\section{METODOLOGIA}

O trabalho foi planejado como uma prática da disciplina Conservação e Preservação de Documentos (2BIB050) do Curso de Biblioteconomia da Universidade Estadual de Londrina-UEL, na qual estavam matriculados vinte e três estudantes.

O exercício de diagnóstico do estado de conservação do acervo foi realizado após as aulas teóricas a respeito dos agentes de deterioração e as consequências danosas ao acervo.

Todos os estudantes da disciplina foram encaminhados para a Biblioteca Setorial de Ciências Humanas $(\mathrm{BSCH})$ para uma atividade prática com quatro horas de duração. 
Maria Aparecida Lopes; Maria Elisabete Catarino; Yara Maria Pereira da Costa Prazeres; Osny Terciotti

Estudo das condições físicas da coleção de livros da área de Direito: um exercício didático

Determinou-se o material a ser avaliado a partir de uma listagem de duzentos e cinco livros das disciplinas de Direito Penal, Direito Processual Penal, Direito do Trabalho e Processo do Trabalho, que fazem parte da bibliografia básica de treze disciplinas do Curso de Direito da Universidade Estadual de Londrina. Essa listagem foi obtida antecipadamente pelos estudantes da disciplina Desenvolvimento de Coleções (2BIB049), integrante do Programa de Formação Complementar do Ensino de Graduação, durante o estudo sobre a cobertura da coleção pertencente à bibliografia básica do referido curso, a qual foi organizada por autores, títulos e o número de chamada de cada obra encontrada no acervo.

Para a realização do exercício de diagnóstico, os estudantes foram divididos em quatro grupos e orientados a localizar os livros nas estantes. Dos 205 (duzentos e cinco) livros constantes da lista, foram encontradas, 66 (sessenta e seis) obras que no momento estavam disponíveis na biblioteca.

Os livros encontrados foram reunidos em uma mesa e avaliados individualmente e de forma padronizada pelos estudantes, por meio de uma ficha de avaliação, adaptada do estudo de KRAMER (anexo). Nessa ficha foi indicado o estado de conservação de cada livro, de acordo com o seu aspecto físico e os principais danos encontrados na obra. Cada estudante avaliou uma média de três livros, sob a orientação da professora da disciplina.

\section{RESULTADOS PARCIAIS}

A análise de dados foi realizada em sala de aula pelos estudantes com a orientação da professora.

Constatou-se que, dentre os principais problemas encontrados nos sessenta e seis livros analisados, destaca-se a falta de cuidado com as obras, pois $97 \%$ dos livros têm anotações em suas páginas, indicando descaso do usuário com o acervo de uso coletivo. 
Maria Aparecida Lopes; Maria Elisabete Catarino; Yara Maria Pereira da Costa Prazeres; Osny Terciotti

Estudo das condições físicas da coleção de livros da área de Direito: um exercício didático

Foi também detectado $81 \%$ dos materiais com poeira e sujidade e $58 \%$ com lombadas danificadas. Em menor proporção, observou-se capas danificadas, uso de fitas adesivas, uso inadequado de cola, excremento de insetos, rasgos e folhas perdidas, dentre outros.

Pelos resultados obtidos, pode-se inferir que a higienização, uma das medidas básicas e de grande relevância no que se refere à conservação preventiva, não está sendo realizada de maneira eficaz.

Outros danos dizem respeito ao manuseio incorreto dos materiais, bem como práticas inadequadas de consertos por meio do uso de colas e fitas adesivas que podem provocar prejuízos irreversíveis ao papel.

Esses dados demonstram a necessidade de um trabalho educativo sobre a importância da conservação, propondo mudanças de comportamento junto aos funcionários com vistas ao uso de procedimentos adequados para sanar problemas que contribuem para a deterioração dos documentos.

O mesmo acontece com relação aos usuários, que também são responsáveis pela degradação dos materiais. Como prevenção, devem ser difundidas normas e medidas educativas que procurem tornar os usuários aliados da biblioteca na manutenção dos acervos.

Outros fatores que podem influenciar no estado de conservação dos livros estão relacionados com a idade dos materiais, a quantidade de uso e até mesmo com a forma de aquisição dos materiais (compra, doação ou permuta).

No entanto, por se tratar de um projeto piloto, executado como exercício preliminar da Disciplina 2BIB050, não foi possível fazer as correlações desses fatores na análise dos dados. Por se tratar de projeto inserido num Programa de Formação Complementar, cuja regulamentação prevê atividades contínuas, outros estudos complementares poderão ser realizados. 
Maria Aparecida Lopes; Maria Elisabete Catarino; Yara Maria Pereira da Costa Prazeres; Osny Terciotti

Estudo das condições físicas da coleção de livros da área de Direito: um exercício didático

\section{CONSIDERAÇÕES PARCIAIS}

O trabalho de diagnóstico sobre o estado de conservação dos livros da bibliografia básica, da área de Direito, existentes na Biblioteca Setorial de Ciências Humanas, realizado pelos estudantes da disciplina Conservação de Documentos do Curso de Biblioteconomia, faz parte do Programa de Formação Complementar no Ensino de Graduação da UEL.

A partir das aulas teóricas sobre o assunto, os estudantes foram levados a vivenciar os problemas que comprometem a integridade física dos acervos documentais e as principais soluções recomendadas para a conservação preventiva do patrimônio cultural e científico das bibliotecas.

Por meio desse instrumento metodológico, os estudantes fizeram o mapeamento da situação, identificando alguns fatores de deterioração provocados principalmente pela falta de higienização, manuseio incorreto e práticas inadequadas utilizadas no conserto dos materiais.

Considerando que as atividades de preservação são reconhecidas como uma das funções de gerenciamento das coleções, os estudantes também tiveram a oportunidade de discutir sobre a importância do estudo para o processo de tomada de decisões no tocante às ações e às medidas preventivas necessárias à conservação dessa coleção analisada.

Nesse sentido, puderam perceber a relevância do diagnóstico como meio imprescindível para o planejamento das diretrizes a serem adotadas em um programa de conservação preventiva de documentos.

Para concluir, vale ressaltar que o bibliotecário, como responsável pela conservação e preservação dos acervos que gerencia, deve conhecer e pesquisar os fatores que provocam a deterioração dos materiais e as formas de combatê-los, a fim de estabelecer políticas e programas adequados à sua realidade. 
Maria Aparecida Lopes; Maria Elisabete Catarino; Yara Maria Pereira da Costa Prazeres; Osny Terciotti

Estudo das condições físicas da coleção de livros da área de Direito: um exercício didático

\section{REFERÊNCIAS}

BECK, I. Manual de conservação de documentos. Rio de Janeiro: Arquivo Nacional, 1997.

BOJANOSKI, Silvana. Oficina: Gestão de preservação de acervos bibliográficos. In: SEMINÁRIO NACIONAL DE BIBLIOTECAS UNIVERSITÁRIAS, 17. 2012. Gramado: SNBU, 2012. Disponível em: $<$ http//:www.snbu2012.com.br/minicursos-e-oficinas/index.php>. Acesso em: 20 set. 2014. (Apresentação de Slides).

CATARINO, M. E. (Coord.). Projeto n.477: a contribuição da gestão de coleções de bibliotecas universitárias para a formação do aluno de biblioteconomia. Londrina: UEL, 2012. Disponível em:

$<$ http://www.sistemasweb.uel.br/index.php?contents=system/prj/ens/inde x.php\&pagina $=$ ens-gry-departamento3.php\&p-cod-centro $=06000000 \& p-$ cod-deptp=06050000>. Acesso em: 19 jul. 2014.

CONWAY, P. Preservação no universo digital. Rio de Janeiro: Projeto conservação preventiva em bibliotecas e arquivos: Arquivo Nacional, 2001.

MAGUETA, Rita de Cassia de Matos. Diagnóstico de conservação fotográfica: uma abordagem arquivística. In: ENCONTRO ESTADUAL DE HISTÓRIA, 11. 2012, Rio Grande. Anais eletrônicos... Rio Grande: FURG, 2012. Disponível em: <ttp//:www.eeh2012.anpuhrs.org.br/resources/anais/18/ 1346114377_ARQUIVO_Artigosubmetido.pdf>. Acesso em: 20 set. 2014.

MOTTA, R. T.; REIS, A. S. dos. Ações de ensino, pesquisa e extensão no LPA-Laboratório de preservação de acervos: um exercício de responsabilidade pública. In: DUARTE, A. B. S.; LOURENÇO, C. de A. (Org.). O carro biblioteca da ECI/UFMG: 38 anos. Belo Horizonte: Rona, 2012, p. 35-47.

OGDEN, S. Métodos de armazenamento e práticas de manuseio. In: BECK, I. (Coord.). Caderno técnico armazenagem e manuseio. Rio de Janeiro: Projeto Conservação Preventiva em Bibliotecas e Arquivos: Arquivo Nacional, 2001a, p. 7-13.

. A proteção contra os danos provocados pela luz. In: BECK, I. (Coord.). Caderno técnico meio ambiente. Rio de Janeiro: Projeto Conservação Preventiva em Bibliotecas e Arquivos: Arquivo Nacional, 2001b, p.13-22. 
Maria Aparecida Lopes; Maria Elisabete Catarino; Yara Maria Pereira da Costa Prazeres; Osny Terciotti

Estudo das condições físicas da coleção de livros da área de Direito: um exercício didático

- Temperatura, umidade relativa do ar, luz e qualidade do ar: diretrizes básicas para a preservação. In: BECK, I. (Coord.). Caderno técnico meio ambiente. Rio de Janeiro: Projeto conservação preventiva em bibliotecas e arquivos: Arquivo Nacional, 2001c, p. 7-11.

SILVA, A. G. da. Procedimentos para aumentar a durabilidade de materiais perecíveis:papel. In: SILVA, R. R. G. da (Org.). Preservação documental: uma mensagem para o futuro. Salvador: EDUFBA, 2012, p. 93-106.

\section{SPINELLI JUNIOR, J. A conservação de acervos bibliográficos \&}

documentais. Rio de Janeiro: Fundação Biblioteca Nacional, 1997.

STOCKER, C. T. Cuidados especiais com acervos bibliográficos: preservação e conservação. In: ABRUNHOSA, J. J. (Org.) Coletânea sobre preservação e conservação de acervos em bibliotecas brasileiras. Nova Friburgo: Êxito Brasil, 2008, p. 53-63.

UNIVERSIDADE ESTADUAL DE LONDRINA. Resolução CEPE $\mathbf{n}$. 142/2012. Da nova regulamentação ao Programa de Ensino de Formação Complementar. Disponível em: $<$ http://www.uel.br/prograd/docs_prograd/resolucoes/2012/resolucao_14 2_12.pdf>. Acesso em: 08 out. 2014.

\section{Title}

Study of Physical Conditions of the Law Books Collection: a didactic exercise

\section{Abstract}

Introduction: This is a record of practical activities with students of the discipline "Documents Preservation (2BIB050)" in the Course of Librarianship from the State University of Londrina-UEL, within the Complementary Training Program in Undergraduate Teaching through the project "The contribution of university libraries management to the formation of Librarianship students".

Objective: To assess the conservation status of the books of the graduation course in Law stored in the Sectorial Library of Humanities / BSCH UEL.

Methodology: The diagnostic exercise was conducted after the theoretical classes on agents of deterioration. Students were referred to the $\mathrm{BSCH}$ to examine the books of the selected subjects, they were given an assessment form adapted from KRAMER study as an instrument.

Results: Partial results indicate that most of the books present dirt and damage on the covers and spines.

Conclusion: It was concluded that the dirt and identified damage are caused by improper handling and lack of hygiene. It is worth highlighting that the librarian, as the one responsible for the conservation and preservation of the collections he/she manages, should know and research about the factors that 
Maria Aparecida Lopes; Maria Elisabete Catarino; Yara Maria Pereira da Costa Prazeres; Osny Terciotti

Estudo das condições físicas da coleção de livros da área de Direito: um exercício didático

cause deterioration of materials and how to fight them in order to establish appropriate policies and programs to their reality.

Keywords: Diagnosis of collections. Collections conservation. Law.

\section{Título}

Estudio de las condiciones físicas de la colección de libros del área de Derecho: un ejercicio didáctico.

\section{Resumen}

Introducción: Relato de actividades prácticas desarrolladas con alumnos de la Asignatura Preservación de Documentos (2BIB050) de la Carrera de Biblioteconomía de la Universidade Estadual de Londrina-UEL, en el ámbito del Programa de Formación Complementaria en la Enseñanza de Graduación a través del proyecto "La contribución de la gestión de bibliotecas universitarias para la formación del alumno de Biblioteconomía".

Objetivo: Evaluar el estado de conservación de los libros de la Carrera de Derecho ubicados en la Biblioteca Sectorial de Ciencias Humanas / BSCH de la UEL.

Metodología: El ejercicio del diagnóstico se ha realizado tras las clases teóricas sobre agentes de deterioro. Los alumnos fueron encaminados a la $\mathrm{BSCH}$ para analizar los libros de las asignaturas seleccionadas teniendo como instrumento una ficha de evaluación adaptada del estudio de KRAMER.

Resultados: Los resultados parciales indican que la mayoría de los libros presenta suciedades y daños en las portadas y lomos.

Conclusiones: Se concluye que las suciedades y daños identificados son provocados por el manoseo incorrecto y la falta de higienización. Resáltese que el bibliotecario, como responsable de la conservación y preservación de los acervos que gerencia, debe conocer e investigar los factores que provocan el deterioro de los materiales y las formas de combatirlos, para que se establezcan políticas y programas adecuados a su realidad.

Palabras clave: Diagnóstico de acervo, Conservación de colecciones.

Derecho.

Recebido em: 15/03/2015

Aceito em: 10/09/2015 